\title{
ChemComm
}

\section{A near-infrared colorimetric fluorescent chemodosimeter for the detection of glutathione in living cells $\dagger$}

50, 1751

Received 23rd October 2013,

Accepted 3rd December 2013

DOI: $10.1039 / c 3 c c 48128 j$

www.rsc.org/chemcomm

\author{
Meng Li, ${ }^{a}$ Xumeng $\mathrm{Wu}^{\mathrm{b}}$ Yao Wang, ${ }^{\mathrm{b}}$ Yongsheng $\mathrm{Li}^{\mathrm{b}}{ }^{\mathrm{b}}$ Weihong $\mathrm{Zhu}^{\star \mathrm{b}}$ and \\ Tony D. James*ab
}

\begin{abstract}
A novel near-infrared (NIR) and colorimetric fluorescent molecular probe based on a dicyanomethylene- $4 \mathrm{H}$-pyran chromophore for the selective detection of glutathione in living cells has been developed. The fluorescence OFF-ON switch is triggered by cleavage of the 2,4-dinitrobenzensulfonyl (DNBS) unit by the interaction with GSH.
\end{abstract}

Biothiols such as glutathione (GSH), cysteine (Cys), and homocysteine (HCys) play a crucial role in maintaining appropriate redox homeostasis in biological systems. ${ }^{1,2} \mathrm{GSH}$, the most abundant cellular thiol, is of great importance in cellular defense against toxins and free radicals. ${ }^{3}$ Changes in the level of GSH concentration have been correlated with various diseases, such as AIDS, leukocyte loss, liver damage, cancer and neurodegenerative diseases. ${ }^{4}$ Therefore, much effort has been devoted to the development of efficient methods for the detection of GSH, which is of significant interest in the fields of chemical, environmental and biological sciences. ${ }^{5,6}$ Many fluorescent sensors for the detection of GSH have been developed based on various fluorophores, including BODIPY, ${ }^{7,8}$ coumarin, ${ }^{9,10}$ rhodamine ${ }^{11,12}$ squaraine, ${ }^{13}$ fluorescein ${ }^{14,15}$ and lanthanide based probes. ${ }^{16}$ However, the chromophore of dicyanomethylene- $4 H$-pyran has seldom been exploited in the detection of thiols. ${ }^{17,18}$ Due to their emission located at the red or near infra-red (NIR) region, (DCM)type derivatives are particularly suitable for application in biological samples, since they produce lower background fluorescence with less scattering, penetrate much deeper into tissues than UV and visible light and cause little damage to living cells. ${ }^{19,20}$ The DCM fluorophore is more photostable than comparable NIR fluorophores such as squaraine and cyanine. Although our previously reported BODIPY fluorophore $^{21}$ is photostable it is much harder to synthesise than the DCM chromophore described here.

\footnotetext{
${ }^{a}$ Department of Chemistry, University of Bath, Claverton Down, Bath, BA2 7AY, UK. E-mail: t.d.james@bath.ac.uk; Fax: +44 (0)1225 386231; Tel: +44 (0)1225 383810

${ }^{b}$ Shanghai Key Laboratory of Functional Materials Chemistry, Key Laboratory for Advanced Materials and Institute of Fine Chemicals, East China University of Science \& Technology, Shanghai 200237, P. R. China. E-mail:whzhu@ecust.edu.cn; Fax: +86-21-6425-2758

$\dagger$ Electronic supplementary information (ESI) available: Detailed procedures, characterization data, and additional plots. See DOI: $10.1039 / \mathrm{c} 3 \mathrm{cc} 48128 \mathrm{j}$
}

A number of sensing mechanisms have been employed for thiol detection, including Michael addition, ${ }^{22}$ to $-\mathrm{CHO}$ attached fluorophores, ${ }^{23}$ and deprotection of 2,4-dinitrobenzenesulfonyl (DNBS), ${ }^{24-26}$ etc. Among them, the DNBS group is especially preferable as an efficient recognition unit for thiols due to its unique sensitivity and high reactivity toward thiolate anions with OFF-ON signalling. With these in mind, we demonstrate the development of a colorimetric and NIR fluorescence turn-on thiol probe 1 (Scheme 1) containing DCM as the fluorophore and DNBS as the fluorescence quencher and recognition moiety. Specifically, we attached a benzene unit to a dicyanopyran moiety, which extended the conjugated system and produced an emission band centred at about $690 \mathrm{~nm}$, thus successfully making the wavelength fall in the desired NIR region. ${ }^{27}$ Particularly, the developed probe 1 exhibits several meritorious features: (i) high sensitivity and selectivity to sulfydryl-containing species; (ii) significant enhancement of NIR fluorescence upon removal of DNBS by GSH; (iii) excellent performance in live cell imaging.

Probe 1 is synthesized by stirring 2-(2-(4-hydroxystyryl)- $4 \mathrm{H}$ chromen-4-ylidene)malononitrile and 2,4-dinitrobenzene-1-sulfonyl chloride in the presence of pyridine at room temperature, to give probe 1 in $45 \%$ yield, which was fully characterized by ${ }^{1} \mathrm{H} \mathrm{NMR}$, ${ }^{13} \mathrm{C}$ NMR, and HRMS, as shown in the ESI $\dagger$ (Fig. S3 and S8). The GSH-promoted specific O-S cleavage of probe 1 liberates $\mathrm{DCPO}^{-}$ with an intramolecular charge transfer (ICT) emission band falling in the desired NIR region at about $690 \mathrm{~nm}$ (Scheme 1).

Initially, UV-vis absorption of probe 1 was investigated. Probe 1 has an intense absorption at $414 \mathrm{~nm}$ (Fig. 1a) in a

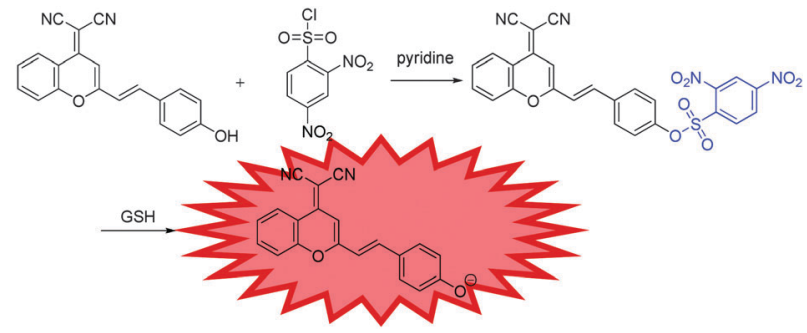

Scheme 1 Synthesis of probe 1 and GSH-promoted release of DCPO- . 

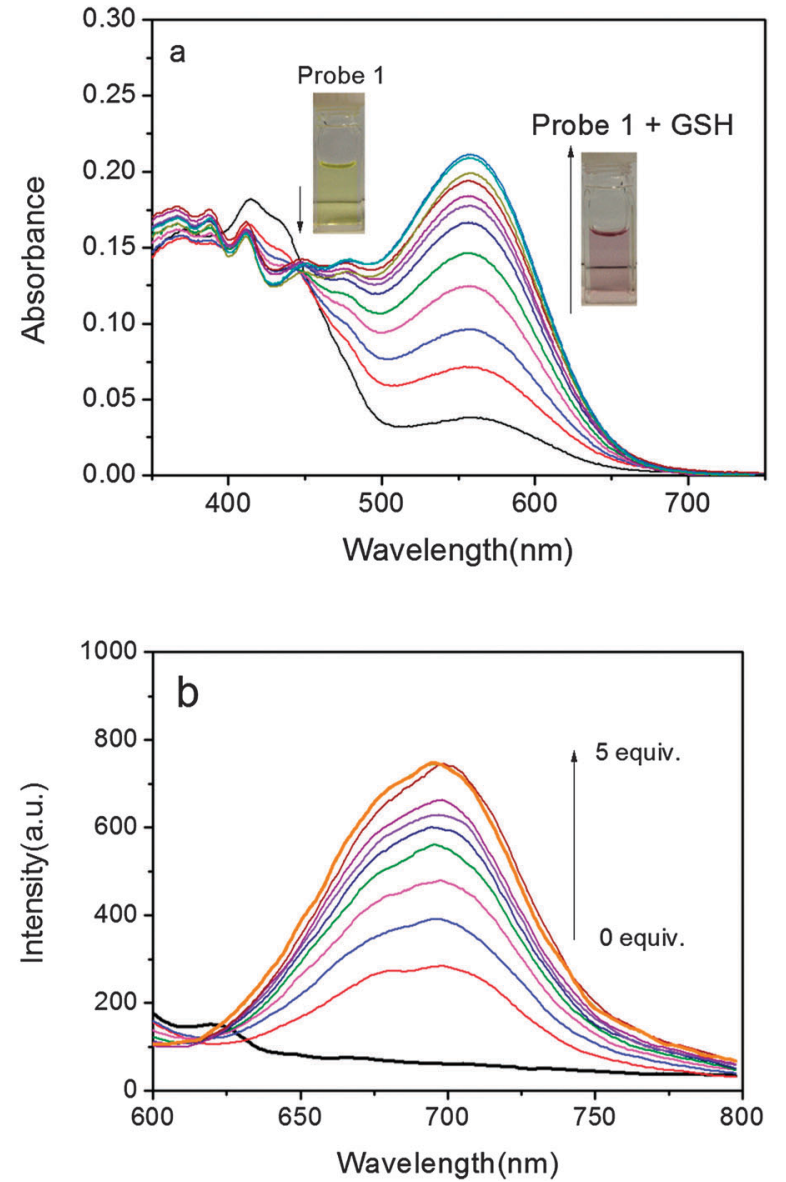

Fig. 1 (a) Absorption spectra of probe $1\left(1 \times 10^{-5} \mathrm{M}\right)$ at different concentrations of GSH $(0,0.1,0.2,0.3,0.4,0.5,0.6,0.7,0.8,1.0,2.0,5.0$ equiv.) in a mixture of DMSO-water $(50: 50, \mathrm{v} / \mathrm{v})$ with a PBS buffer solution (10 $\mathrm{mM}, \mathrm{pH}=7.4)$. Insets: colour changes of probe 1 upon addition of GSH (5 equiv.). (b) Fluorescence spectra of probe $1\left(1 \times 10^{-5} \mathrm{M}\right)$ at different concentrations of GSH when excited at $560 \mathrm{~nm}$.

DMSO-PBS buffer solution $(\mathrm{pH}=7.4,50 / 50, \mathrm{v} / \mathrm{v})$ at $37{ }^{\circ} \mathrm{C}$. Upon the addition of GSH, the colour of the solution turned from slight yellow to pink, which was clearly recognizable by the naked eyes. At the same time, a concomitant increase of a new absorption band at $560 \mathrm{~nm}$ was observed with an isosbestic point at $446 \mathrm{~nm}$. As illustrated in Fig. 1a, due to the specific O-S cleavage, a distinct $146 \mathrm{~nm}$ red shift in absorbance was observed (Scheme 1). Since the phenolate group is a much stronger electron donor than the sulfonate group, the ICT efficiency should be significantly enhanced by the interaction of probe 1 with GSH and thus shifting the absorption to a longer wavelength.

Subsequently, the fluorescence spectra of probe $\mathbf{1}$ in the absence and presence of GSH were recorded. The probe alone exhibits almost no emission when excited at $560 \mathrm{~nm}$ and no distinct variations are observed over time, suggesting that probe $\mathbf{1}$ is stable and has no tendency to convert into $\mathrm{DCPO}^{-}$under the measurement conditions. In the presence of $\mathrm{GSH}$, however, fluorescence at $690 \mathrm{~nm}$ was dramatically enhanced (Fig. 1b) $\$$ The departure of the electronwithdrawing DNBS moiety via a GSH-induced O-S bond cleavage releases $\mathrm{DCPO}^{-}$, which possesses strong ICT and induces a turn-on NIR fluorescence response with a high off/on ratio. On the other hand, the large Stokes shift of $\sim 130 \mathrm{~nm}$ is also conducive to decrease the background fluorescence, enhancing the signal fidelity. Moreover, the response rate of $\mathbf{1}$ to GSH was tested by time-course fluorescence measurements. In the presence of GSH, the fluorescence intensity at $690 \mathrm{~nm}$ increases gradually and reaches a plateau after about $5 \mathrm{~min}$ (Fig. S1†), indicating the completion of the reaction.

To evaluate the selectivity of the developed probe $\mathbf{1}$ for sulfydrylcontaining species, a series of amino acids were examined. Not surprisingly, except for GSH, probe 1 shows similar response to other sulfydryl-containing compounds such as cysteine, homocysteine, dithiothreitol, while no measurable fluorescence enhancement could be triggered by the treatment of other amino acids (Fig. 2). In fact, the plausible disturbance of DTT, Cys and HCy can be neglected due to their relatively low concentration in biological systems. ${ }^{28}$ To gain insight into the sensing mechanism, mass spectral analysis was further performed. The reaction between probe $\mathbf{1}$ and GSH that occurred by the cleavage of $\mathrm{O}-\mathrm{S}$ with the release of $\mathrm{DCPO}^{-}$was confirmed by the appearance of the peak at 311.0828 in the mass spectrum (Fig. S $3 \dagger$ ). Furthermore, the fluorescence intensity at $690 \mathrm{~nm}$ shows a linear relationship $(R=0.98)$ with GSH concentrations from 1 to $10 \mu \mathrm{M}$, and the detection limit was evaluated to be $1.8 \times 10^{-8} \mathrm{M}$, indicating that probe 1 is particularly sensitive to the detection of GSH (Fig. S2 $\dagger$ ).

The effect of $\mathrm{pH}$ on the photophysical properties of DCPO and probe 1 was then investigated (Fig. $\mathrm{S} 4 \dagger$ and $\mathrm{S} 6 \dagger$ ). As illustrated, we believe that the probe is quite stable in aqueous media up to $\mathrm{pH} 8$, whereas DCPO displays a corresponding fluorescence enhancement in the $\mathrm{pH}$ range of 5.7-8.0, which clearly demonstrates that the fluorescence response of probe $\mathbf{1}$ in the physiological $\mathrm{pH}$ range is due to the presence of thiols and verifies the generation of $\mathrm{DCPO}^{-}$ after treatment with GSH. Therefore, we decided to employ the probe for fluorescent imaging of cellular thiols. The $\mathrm{p} K_{\mathrm{a}}$ of DCPO was calculated to be 8.70 (Fig. S5†).

The ability of probe 1 to detect GSH in HeLa cells was examined using confocal fluorescence microscopy (Fig. 3). HeLa cells were

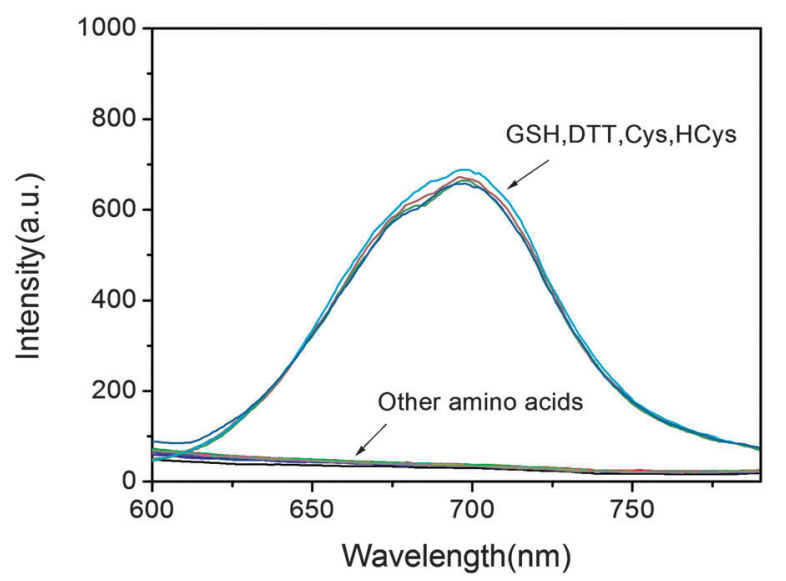

Fig. 2 Fluorescence spectra of probe 1 in the absence and presence of various amino acids and GSH, DTT, Cys, HCys in a mixture of DMSO-water $(50: 50, v / v)$ with a PBS buffer solution $(10 \mathrm{mM}, \mathrm{pH}=7.4)$. amino acids are arginine, threonine, serine, isoleucine, asparaginic acid, sarcosine, valine, glutamine, tryptophan, glutamic acid, proline, leucine, histidine, glycine, phenylalanine, alanine, asparagine, methionine. 


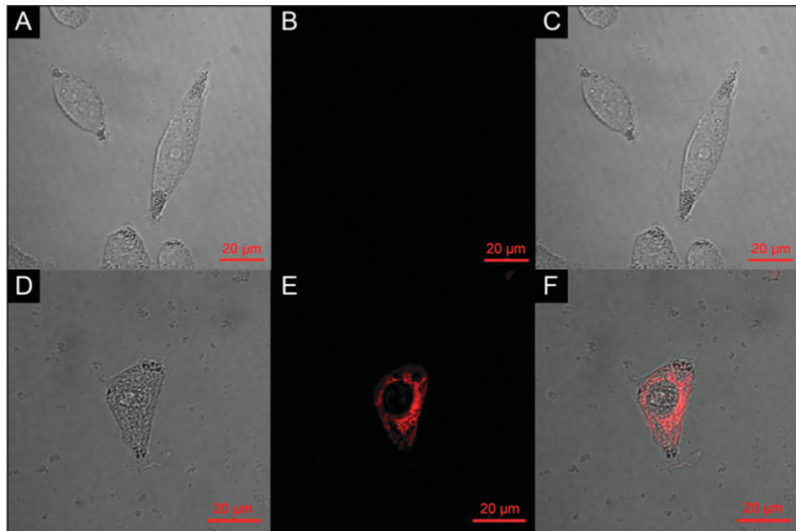

Fig. 3 Confocal fluorescence images in HeLa cells: (top) (A-C) cell incubated without probe 1; (bottom) (D-F) cell incubated with probe 1 $(20 \mu \mathrm{M})$ for $0.5 \mathrm{~h}$. Emission was collected at $660-740 \mathrm{~nm}$ upon excitation at $488 \mathrm{~nm}$. Bright field ( $A$ and $D$ ), fluorescence ( $B$ and $E$ ) and overlap field ( $C$ and F).

cultured in RPMI 1640 supplemented with 10\% FCS and then incubated with $10 \%$ fetal bovine serum followed by $20 \mu \mathrm{M}$ of probe 1 in PBS for $30 \mathrm{~min}$. 2.5\% DMSO was used in the cell culture process. Bright-field measurements confirmed that the cells after treatment with probe $\mathbf{1}$ were viable throughout the imaging experiments, indicating the superior biocompatibility of probe 1. After the co-culture with probe 1, remarkable intracellular NIR fluorescence could be detected. The overlay of fluorescence and bright-field images indicates that the fluorescence localizes mainly in the cytosol, indicative of the subcellular distribution of GSH and excellent membrane permeability of probe 1 . In contrast, cells that are not treated with probe 1 display nearly no fluorescence, verifying the fluorescence source of the 1-treated cells (Fig. 3B). Moreover, the images also indicate that the probe has low toxicity since the cellular morphology is maintained. With this bioimaging experiment we have demonstrated the potential of probe $\mathbf{1}$ for the imaging of GSH in living cells.

In conclusion, we have designed and synthesized a NIR fluorescent chemodosimeter for GSH based on a DCM framework, showing a subcellular distribution of GSH and good cell membrane permeability. The fluorescence enhancement mechanism is based on the cleavage of DNBS from the fluorophore by GSH, which switches the dark excited state to an emissive excited state. Probe 1 displays a colour change from yellow to pink upon addition of GSH, and thus can serve as a "naked-eye" probe for GSH. Furthermore, we have also demonstrated that the probe can be used for the fluorescent imaging of cellular thiols. Since our DCM based system is very easy to prepare and has a long emission wavelength and high photo-stability, we believe that it will become the probe of choice for biological applications.

T.D.J and M.L. are grateful for financial support from China Scholarship Council (CSC) and University of Bath Full Fees Scholarship. The Catalysis And Sensing for our Environment
(CASE) network is thanked for research exchange opportunities. W.H.Z. is grateful for financial support from the Oriental Scholarship, and the Fundamental Research Funds for the Central Universities (WK1013002). T.D.J thanks ECUST for a guest professorship. T.D.J. also thanks the Royal Society for support.

\section{Notes and references}

$\$$ When probe 1 is excited at the isosbestic point (446 nm, Fig. S7†) a fluorescence increase at $581 \mathrm{~nm}$ is observed which is ascribed to release of the DNBS quenching group. Conversely, when probe $\mathbf{1}$ is excited at $560 \mathrm{~nm}$ the large observed increase in fluorescence at $690 \mathrm{~nm}$ is due to the fluorescence of the $\mathrm{DCPO}^{-}$produced upon cleavage of DNBS from probe 1 .

1 H. Refsum, A. D. Smith, P. M. Ueland, E. Nexo, R. Clarke, J. McPartlin, C. Johnston, F. Engbaek, J. Schneede and C. McPartlin, Clin. Chem., 2004, 50, 3-32.

2 Z. A. Wood, E. Schröder, J. Robin Harris and L. B. Poole, Trends Biochem. Sci., 2003, 28, 32-40.

3 A. Pastore, F. Piemonte, M. Locatelli, A. L. Russo, L. M. Gaeta, G. Tozzi and G. Federici, Clin. Chem., 2001, 47, 1467-1469.

4 H. Tapiero, D. Townsend and K. Tew, Biomed. Pharmacother., 2003, 57, 134-144.

5 D. Zhai, S.-C. Lee, S.-W. Yun and Y.-T. Chang, Chem. Commun., 2013, 49, 7207-7209.

6 X. Chen, Y. Zhou, X. Peng and J. Yoon, Chem. Soc. Rev., 2010, 39, 2120-2135.

7 X. Li, S. Qian, Q. He, B. Yang, J. Li and Y. Hu, Org. Biomol. Chem., 2010, 8, 3627-3630.

8 L.-Y. Niu, Y.-S. Guan, Y.-Z. Chen, L.-Z. Wu, C.-H. Tung and Q.-Z. Yang, J. Am. Chem. Soc., 2012, 134, 18928-18931.

9 G.-J. Kim, K. Lee, H. Kwon and H.-J. Kim, Org. Lett., 2011, 13, 2799-2801.

10 W. Lin, L. Yuan, Z. Cao, Y. Feng and L. Long, Chem.-Eur. J., 2009, 15, 5096-5103.

11 W. Lin, L. Long and W. Tan, Chem. Commun., 2010, 46, 1503-1505.

12 B. Tang, Y. Xing, P. Li, N. Zhang, F. Yu and G. Yang, J. Am. Chem. Soc., 2007, 129, 11666-11667.

13 S. Sreejith, K. P. Divya and A. Ajayaghosh, Angew. Chem., 2008, 120, 8001-8005.

14 H. Y. Shiu, H. C. Chong, Y. C. Leung, M. K. Wong and C. M. Che, Chem.-Eur. J., 2010, 16, 3308-3313.

15 X. Chen, S.-K. Ko, M. J. Kim, I. Shin and J. Yoon, Chem. Commun., 2010, 46, 2751-2753.

16 B. K. McMahon and T. Gunnlaugsson, J. Am. Chem. Soc., 2012, 134, 10725-10728.

17 J. Cao, C. Zhao and W. Zhu, Tetrahedron Lett., 2012, 53, 2107-2110.

18 G. Kwak, H. Kim, I.-K. Kang and S.-H. Kim, Macromolecules, 2009, 42, 1733-1738.

19 Z. Guo, W. Zhu and H. Tian, Chem. Commun., 2012, 48, 6073-6084. 20 W. Zhu, X. Huang, Z. Guo, X. Wu, H. Yu and H. Tian, Chem. Commun., 2012, 48, 1784-1786.

21 J. Shao, H. Sun, H. Guo, S. Ji, J. Zhao, W. Wu, X. Yuan, C. Zhang and T. D. James, Chem. Sci., 2012, 3, 1049-1061.

22 N. Zhao, Y.-H. Wu, L.-X. Shi, Q.-P. Lin and Z.-N. Chen, Dalton Trans., 2010, 39, 8288-8295.

23 R. Zhang, X. Yu, Z. Ye, G. Wang, W. Zhang and J. Yuan, Inorg. Chem., 2010, 49, 7898-7903.

24 S. Ji, H. Guo, X. Yuan, X. Li, H. Ding, P. Gao, C. Zhao, W. Wu, W. Wu and J. Zhao, Org. Lett., 2010, 12, 2876-2879.

25 J. Bouffard, Y. Kim, T. M. Swager, R. Weissleder and S. A. Hilderbrand, Org. Lett., 2008, 10, 37-40.

26 S.-P. Wang, W.-J. Deng, D. Sun, M. Yan, H. Zheng and J.-G. Xu, Org. Biomol. Chem., 2009, 7, 4017-4020.

27 X. Huang, Z. Guo, W. Zhu, Y. Xie and H. Tian, Chem. Commun., 2008, 5143-5145.

28 L. Ducry and B. Stump, Bioconjugate Chem., 2009, 21, 5-13. 